\title{
LA CORTE COSTITUZIONALE
}

\author{
di Mario Dogliani e Ilenia Massa Pinto
}

1. Il fascicolo al quale queste pagine sono destinate avvia una riflessione su un concetto inusuale nella teoria costituzionale: il concetto di frattura, intendendo con esso "i fatti che hanno provocato una rottura non recuperabile rispetto al passato ... che segnano una cesura non recuperata ... un profondo cambiamento di tendenza". Fatti che si manifestano "nei processi di modernizzazione, costituzionalizzazione, ed europeizzazione" e che non riguardano "necessariamente tutto il sistema politico, economico e istituzionale" potendo riguardare anche solo uno o più aspetti della vita del Paese". Dunque un concetto del tutto difforme da quello - presente nella meno recente dogmatica giuspubblicistica - di "rottura della Costituzione". Con questa espressione si intende(va) fare riferimento a quelle "altre leggi costituzionali", previste dall'art. 138 Cost., che eventualmente facciano "venir meno il vigore di norme costituzionali, con riferimento ad un caso singolo o anche per una generalità di casi regolati, in modo da costituire un'eccezione rispetto alla regola generale vigente per gli altri casi": norme derogatorie singolari, dunque, come quelle contenute nello stesso testo costituzionale, alle disposizioni XII e XIII; e che, come queste, "consentite nei limiti dello scopo supremo di preservazione dei valori fondamentali inerenti al tipo proprio dell'ordinamento vigente" 1 . Il tema dovrebbe allargarsi alle convenzioni sulla Costituzione e alle modifiche consuetudinarie e convenzionali; ma ci porterebbe fuori strada. Se continuassimo a chiederci: che cosa è che si frattura? saremmo indotti - per questa via - a chiederci se sia la stessa Costituzione, che perde in modo irrecuperabile la propria effettività e la pro-

1 C. Mortati, Istituzioni di diritto pubblico, Padova, CEDAM, 1976, tomo II, pp. 12371238. 
pria legittimazione, e apre nuovi processi costituenti. Oppure se sia il tipo di legittimazione originaria, al quale se ne sostituisce uno parzialmente nuovo. Oppure se sia il passaggio di fatto dalla concezione rigida della Costituzione come "un tutto" ad una concezione sostanzialmente "flessibile" o, addirittura, ad una totalmente sovrastrutturale, come quella sottesa al brocardo forma regiminis mutata, non mutatur ipsa civitas.

Forse il modo più semplice di risolvere il problema è di abbandonare il terreno della teoria costituzionale e considerare il concetto di frattura come un concetto di natura puramente storiografica che riguarda la cultura costituzionale "vivente" e che va usata (almeno inizialmente) a fini descrittivi. L'oggetto della frattura non è dunque (direttamente) la validità o l'effettività della Costituzione, ma il modo d'intenderla: la cultura costituzionale diffusa. Altro sarà la valutazione degli effetti che la frattura potrà aver prodotto sulla Costituzione. Effetti che potranno oscillare tra la negazione della sua continuità e portare alla instaurazione di una nuova Costituzione (scelta repubblicana) o segnando gli eventi più significativi della successiva storia costituzionale. Il termine frattura può dunque riferirsi o a eventi storici che sono in sé, nel loro puntuale verificarsi, sintomi dell' affermazione vittoriosa di una diversa teoria dello Stato (instaurazione della repubblica), oppure dell'introduzione nel testo costituzionale di discipline sintomo di una diversa teoria dello Stato destinate ad avere successivi e rilevanti sviluppi.

2. Per quanto riguarda l'entrata "in funzione" della Corte costituzionale è qui inutile ripercorrere le note vicende svoltesi in seno al1'Assemblea Costituente: dalla comune opzione per una Costituzione rigida alle differenze manifestatesi a proposito dell'introduzione della giustizia costituzionale. Fortemente voluta dal gruppo democristiano (attento all'equilibrio del futuro assetto, e cioè alla possibilità di imbrigliare temuti "eccessi" di eventuali governi delle sinistre) e nettamente avversata dal partito comunista, timoroso che il governo dei giudici si sovrapponesse al governo del popolo, quale sarebbe stato reso possibile da un governo parlamentare puro. Il successivo mutamento di opinione del partito comunista fu dovuto non ad una clamorosa (opportunistica) svolta, come ancora oggi si tende a dire, ma 
alla rottura dell'unità ciellenista, con l'esclusione dei socialcomunisti dal IV Governo De Gasperi (maggio 1947-maggio 1948), all'inizio della guerra fredda - nei tesissimi ultimi mesi del 1947 - e all'anticomunismo sempre più eccitato (si preparava il contesto che avrebbe portato il $1^{\circ}$ luglio 1949 il Sant'Uffizio a decretare che i cristiani che professano la dottrina comunista materialista e anticristiana, e soprattutto coloro che la difendono e la propagano, incorrono ipso facto nella scomunica riservata alla Sede Apostolica, in quanto apostati della fede cattolica).

Questo clima, così diverso da quello che aveva retto i governi del CLN, portò a mutare la visione costituzionale di tale partito. Non bisogna infatti dimenticare che la approvazione della Costituzione avrebbe dovuto rappresentare - in tale visione - l'avvio di una forma di governo stabilmente imperniata sulla collaborazione dei tre partiti popolari (DC, PCI, PSI), e che dunque si sarebbe avvicinata, di fatto, più alla forma di governo direttoriale che a quella parlamentare classica.

3. In ogni caso, grazie alla tenuta dell" "arco costituzionale" (i partiti che erano stati uniti nel CLN) nella Assemblea Costituente, dopo la rottura a livello governativo, la giustizia costituzionale fu introdotta nella Carta. Con ciò si verificava l'inizio della frattura. Si apriva la sua primissima fase: una frattura ancora incerta nei suoi esiti (è infatti parte essenziale della "costituzione trappola" in cui Scelba disse che non sarebbe mai caduto) e nelle sue conseguenze effettive, ma comunque sintomo del fatto che una nuova teoria dello Stato era stata recepita dalla cultura dei costituenti, e che il dogma della sovranità della legge, quale atto di chiusura dell'intero ordinamento, era finito.

In una seconda fase la frattura ha assunto effettività attraverso l'attività politico- parlamentare che, sfociata nella 1.11 marzo 1953, n. 8, ne ha consentito il dispiegarsi sull'ordinamento legislativo; e soprattutto attraverso la prima sentenza emanata dalla Corte, la n. 1/56, che ha reso più chiari i termini della sottomissione alla Costituzione dell'ordinamento legislativo.

Se la prima fase segna la fine della sovranità della legge, la seconda segna l'insieme delle vicende in cui la legge ha dovuto cedere il diritto all'ultima parola.

Questa seconda fase va osservata almeno da due diversi punti di 
vista: quello del merito delle pronunce in relazione al caso dedotto in giudizio; e quello delle pronunce in cui la Corte ha definito i caratteri e i limiti della sindacabilità della legge attraverso l'invenzione dei propri strumenti, con ciò determinando la propria posizione nei confronti del Parlamento e dei giudici. Non si tratta di due ambiti separati e indipendenti, ma è esaminando questo secondo genere di pronunce che emerge il disegno complessivo della frattura, che può essere così, anticipatamente, riassunto: a) nella scelta di uscire dallo schema del legislatore negativo, b) nella auto-assunzione del potere di determinare il parametro costituzionale integrandolo con riferimenti implicitamente racchiusi nella "Costituzione come un tutto", c) di valorizzare la scelta della instaurazione incidentale, intendendola come scelta che impone alla Corte di fornire indicazioni per la soddisfazione della pretesa dedotta in giudizio, orientando o vincolando il giudice nella individuazione della norma di legge da applicare in conseguenza della dichiarazione di incostituzionalità (esplicita o no). In relazione a quest'ultimo punto occorre però precisare che alcuni recenti sviluppi (dovuti alla ritenuta necessità di eliminare le "zone d'ombra", cioè quelle parti della legislazione che il metodo d'instaurazione incidentale non consente di portare al giudizio della Corte), parrebbero avvicinarsi più al modello del ricorso diretto che non a quello del ricorso in via incidentale (v. sentenze n. 1 del 2014 e n. 35 del 2017). Similmente viene spezzato il rapporto tra giudizio a quo e giudizio di incostituzionalità nei casi in cui viene eluso il requisito della rilevanza della questione (v. ad esempio la sentenza n. 10 del 2015).

4. Assumendo il punto di vista storiografico prima accennato, le principali vicende nelle quali la Corte ha definito i caratteri e i limiti della sindacabilità della legge, e dunque i propri strumenti e la propria posizione e, di riflesso, quella del Parlamento e dei giudici, possono essere così individuate:

a) lo svuotamento teorico della distinzione tra political questions e non political questions ${ }^{2}$ (perché tale distinzione, e il conseguente

2 Art. 28, 1.11 marzo 1953, n. 87: il controllo di legittimità della Corte costituzionale su una legge o un atto avente forza di legge esclude ogni valutazione di natura politica e ogni sindacato sull'uso del potere discrezionale del Parlamento. 
divieto di affrontare le prime, impedirebbe qualunque sindacato della legge sulla base del principio d'uguaglianza formale, dovendosi stabilire, in base ad esso, se il legislatore abbia considerato uguali situazioni disuguali, o disuguali situazioni uguali: giudizi di evidente natura politica) e la conseguente sostituzione dell'antitesi political/non political con quella ragionevole/non ragionevole. Antitesi che non poteva non generare la produzione di una occasionale, e dunque imprevedibile, giurisprudenza.

b) L'utilizzabilità delle norme programmatiche al fine di sindacare una legge alla luce del nucleo (contenuto minimo essenziale) di una norma costituzionale che si limita a prevedere la necessità di una futura concretizzazione legislativa.

c) L'accettazione della dottrina secondo la quale il catalogo dei diritti inviolabili ex art. 2 Cost. è aperto, al fine di sindacare una legge anche in assenza di una norma costituzionale esplicita che sancisca un diritto.

d) Il rifiuto della posizione secondo cui la Corte non può ricavare dall'ordinamento legislativo norme implicite (per colmare la lacuna conseguente alla dichiarazione di incostituzionalità) perché, nel caso in cui tale norma implicita sia presente, spetta a tutti i giudici comuni ricavarla mentre, nel caso in cui tale norma implicita non sia presente, spetta al legislatore crearla; e l'assunzione della contraria posizione per cui la Corte stessa può colmare la lacuna facendo ricorso a sentenze-legge.

e) L'accettazione della posizione secondo cui la legge, soprattutto su impulso dei giudici nazionali, è sostanzialmente sindacabile dalla Corte di Giustizia dell'Unione Europea, e dunque anche alla luce di parametri non tratti dalla Costituzione dello Stato. Posizione, questa, in riferimento alla quale la Corte sta inviando segnali contradditori, che indicano il tentativo di riaccentrare il giudizio sulla costituzionalità delle leggi ponendo un argine al ricorso dei giudici nazionali alla Corte europea di Giustizia, evitando così l'aggiramento della Corte stessa e contestualmente quello della legge da parte dei giudici (v. sentenze n. 269 del 2017, n. 20, n. 63 e n. 112 del 2019 e ord. n. 117 del 2019). Questo riaccentramento ha ripercussioni tanto sul rapporto tra ordinamenti, perché protegge i princìpi fondamentali del nostro ordinamento costituzionale impedendone l'indebolimento ad opera di quelli sovranazionali, se 
non addirittura la tralatizia sostituzione degli uni con gli altri; quanto sul rapporto tra giudice e Costituzione, perché impedisce che il giudice vanifichi la legge attraverso la prospettazione di un parametro evanescente che mantiene solo più un debole legame con la Costituzione italiana.

f) La condivisione dell'idea secondo cui l'attività normativa dell'Unione Europea, le giurisprudenze delle Corti di Lussemburgo e di Strasburgo, e soprattutto, il dialogo formale e informale tra le Corti di ultima istanza, abbiano avviato un processo di formazione di un diritto costituzionale europeo comune, o addirittura cosmopolita.

g) La condivisione dell'idea secondo cui tale diritto costituzionale comune o cosmopolita è l'esito di processi logici, dovuti alla forza generalizzante dei suoi princìpi base (derivanti più che dalle identità costituzionali - positive - degli Stati, dalla loro natura - "spirituale" di "valori"), e non l'esito di processi volontari, politicamente guidati.

5. Fin qui uno sguardo retrospettivo sulle innovazioni che la Corte stessa ha elaborato.

Volendo tentare uno sguardo sintetico sulla frattura che attraverso queste innovazioni la Corte ha realizzato, si deve innanzi tutto precisare che il modello costituzionale - compresa la disciplina contenuta nella legge n. 53/1987 - è molto scarno ed incerto. Inevitabilmente si sarebbe prestato a sviluppi imprevedibili. Le strade che la Corte aveva davanti erano due: quella dell'autolimitazione, in nome del rispetto, fino al massimo del possibile, della sovranità parlamentare "residua", oppure quella di adottare una giurisprudenza di stimolo nei confronti del potere politico fino ad invaderne il campo.

Se avesse intrapreso la prima strada, la Corte avrebbe dovuto limitarsi a controllare le sole violazioni più dirette e manifeste delle norme costituzionali scritte e non troppo sotto-determinate (e cioè rispettare quanto resta - dopo l'introduzione della giustizia costituzionale - del divieto di sindacare la discrezionalità politica del legislatore). Ma così la Corte non ha proceduto. Essa piuttosto ha esteso quanto più possibile, e spesso anche oltre (come nel caso delle sentenze legge non "a rime obbligate"), il suo controllo sulle scelte discrezionali del legislatore. Questa estensione è stata realizzata in due modi apparentemente contradditori: da un lato, servendo le esigenze di giustizia dei casi con- 
creti colte dalla Corte stessa, e dunque sfruttando al massimo la ratio del modello di instaurazione in via incidentale; dall'altro allontanandosi da tale modello fino a dichiarare ammissibili questioni proposte in modo sostanzialmente diretto. Nel primo caso la Corte ha esercitato la sua funzione politica praticando il massimo della pressione sulla legislazione ordinaria, facendo così emergere in modo sempre più esplicito il suo dualismo rispetto al Parlamento; nel secondo fa "silenzio, ed arbitro s'asside" direttamente fra due confliggenti opzioni politiche (ad esempio: rappresentatività vs governabilità nella legge elettorale, sentenze n. 1 del 2014 e n. 35 del 2017).

In entrambi i casi la Corte ha fatto ricorso a tecniche decisorie in cui un ruolo centrale è sempre assunto dal principio di ragionevolezza (il cui parametro testuale è l'art. 3, comma 1, Cost.), ma con due prospettive diverse, che possono apparire paradossali. Se la questione d'incostituzionalità è dominata dalla preoccupazione della Corte per le conseguenze che la sua decisione avrà sulla soluzione del caso concreto, essa sarà il più possibile attenta alle specificità di quest'ultimo. Ma questa attenzione accentuerà la riconducibilità del giudizio di ragionevolezza a quello sulla "natura delle cose". E così il più artificiale dei principi costituzionali - il principio di uguaglianza - finisce con l'essere usato per definire ciò che è più "naturale". Se invece la questione di incostituzionalità è dominata dalla preoccupazione della Corte di arbitrare tra due astratte opzioni politiche, il peso del testo costituzionale sarà maggiore perché la Corte sarà inevitabilmente portata $\mathrm{a}$ far prevalere la soluzione più rispettosa del suo impianto originario, più artificiale, perché è pur sempre da esso che trae la sua legittimazione. Ciò non toglie che anche in questi casi la Corte non faccia uso delle più audaci auto-innovazioni (come nel caso della introduzione della preferenza unica con la sentenza n. 1 del 2014).

6. Una ulteriore constatazione che può concorrere a chiarire la portata della frattura provocata dalla entrata in funzione della Corte costituzionale è che essa ha svolto un ruolo determinante nel disvelamento della componente volontaristica nella interpretazione del diritto scritto. Componente da sempre nota, ma temuta. Mentre lo sforzo dei giuristi, secondo il positivismo analitico (prima dominante in Italia), avrebbe dovuto tendere alla precisazione scientifica dei concetti giuridici, assi- 
milandoli a quelli matematici, la Corte ha esteso al massimo il ruolo dell'interprete richiamando la sua "libertà non limitabile" nella scelta dei criteri ermeneutici, non gerarchizzati, da utilizzare per determinare il significato degli enunciati. La Corte ha, per così dire, messo l'interprete, solo, davanti alla disposizione (costituzionale o ordinaria), vincolato unicamente dai limiti della elasticità dei segni linguistici; si potrebbe dire: dalla loro portanza.

L'esempio della Corte costituzionale e il suo magistero nei confronti dei giudici hanno segnato la vittoria della concezione kelseniana della interpretazione, intesa come atto volitivo (entro il quadro rappresentato dai limiti del segno linguistico) più che come atto cognitivo, come avrebbe voluto la scienza del diritto di matrice positivistica. Il profilo cognitivo dell'interpretazione è limitato alla conoscenza dei confini del segno linguistico. La Corte costituzionale ha liberato i giudici dai vincoli della scienza giuridica. Non operano più "dentro" un quadro concettuale sostantivo inteso, nella sua organicità, come una scienza.

7. Queste considerazioni sul carattere misto - cognitivo-volitivo, con prevalenza di quest'ultimo - della interpretazione della Costituzione avvicinano alla soluzione del problema centrale che dobbiamo risolvere: qual è il nucleo della frattura? $\mathrm{O}$, se si vuole, qual è la "frattura madre" dalla quale tutte le altre derivano?

Ex post si può constatare che la previsione in Costituzione di "principi di giustizia" espressi con formule sotto-determinate, sintomi di altrettanti compromessi intervenuti tra forze politiche conflittuali in sede costituente, non poteva non avere come conseguenza quella trasformazione costituzionale tacita che ha riconosciuto alla giurisdizione costituzionale un carattere sostanzialmente rappresentativo. Si potrebbe dire che la Corte ha assorbito quella quota del rapporto rappresentativo che non deriva dal basso, ma che procede dall'alto. Fare derivare la rappresentanza solo dal basso è una concezione volgare. Rappresentanza è infatti prima di tutto rendere presente, capace di parlare e di agire quel soggetto la cui assenza è insopportabile (nella Chiesa, Dio; in una democrazia, il demos). Abbiamo già accennato al fatto che la sottoposizione della legge a un giudizio comporta la fine della sua sovranità (superiorem non recognoscens) perché il Parlamento non rappresenta 
più in via definitiva la volontà popolare "ultima", dal momento che in questa volontà deve essere compresa anche quella costituente-costituzionale. Questo fenomeno avrebbe potuto avere, però, delle dimensioni più contenute, tali da non far divenire un luogo comune il fatto che la legge è stata "detronizzata". Supra abbiamo illustrato le condizioni alle quali ciò avrebbe potuto avvenire.

Ma per meglio chiarire questo punto - che potremmo descrivere come la traslazione della funzione unificante-rappresentativa dal Parlamento agli organi di garanzia (perché è viva vox constitutionis anche il Presidente della Repubblica) - è opportuno mettere in rilievo quale sia stata la "frattura madre", da cui tutte le altre sono derivate come anelli di una catena. A parte quella consistita nella previsione stessa della giustizia costituzionale, tale "frattura madre" si è verificata quando i diritti sono stati considerati quali oggetti ricettizi dell'art. 2 Cost., anziché quali significati ricavati dal diritto oggettivo: tutte le altre fratture sono sviluppi e conseguenze di questa. Secondo il positivismo giuridico i diritti soggettivi sono "stenografie" che raccolgono l'esito, per il soggetto, della presenza, nel diritto oggettivo, di determinate discipline (la disciplina del Servizio sanitario nazionale consente di dire che gli individui viventi sul territorio della Repubblica sono titolari del diritto alla salute, e così le discipline a tutela della proprietà...).

Questo "sganciamento" del diritto soggettivo dal diritto oggettivo, e questa sua sovra-ordinazione, può essere spiegata, restando all'interno del diritto positivo, in più modi: a) seguendo una teoria che faccia discendere la validità delle costituzioni nazionali dal diritto internazionale, per cui i diritti stabiliti dal diritto internazionale o sovranazionale oggettivo diventano implicitamente e necessariamente oggetti ricettizi del diritto costituzionale interno, che solo così, stabilendo questo collegamento, mantiene la propria validità; b) facendo leva sulle teorie del rinvio alle fonti costituite da fatti normativi. Su questo torneremo più avanti.

8. Quando è avventa questa frattura? Nella sua prima sentenza, la notissima n. 1 del 1956, la Corte, pur discostandosi dalla teoria, sostenuta anche dalla Corte di Cassazione, delle norme costituzionali di principio come norme programmatiche e non immediatamente precettive nei 
confronti dei soggetti diversi dal legislatore, sembrava concepire il proprio ruolo come quello di un legislatore negativo chiamato a smantellare norme - anche anteriori alla entrata in vigore della Costituzione che, in qualche modo, disciplinando un diritto, eccedono nella previsione dei pur necessari limiti che a ogni diritto derivano dal suo essere parte di un sistema di esigenze costituzionali: "la nota distinzione fra norme precettive e norme programmatiche può essere bensì determinante per decidere della abrogazione o meno di una legge, ma non è decisiva nei giudizi di legittimità costituzionale, potendo la illegittimità costituzionale di una legge derivare, in determinati casi, anche dalla sua non conciliabilità con norme che si dicono programmatiche, tanto più che in questa categoria sogliono essere comprese norme costituzionali di contenuto diverso: da quelle che si limitano a tracciare programmi generici di futura ed incerta attuazione, perché subordinata al verificarsi di situazioni che la consentano, a norme dove il programma, se così si voglia denominarlo, ha concretezza che non può non vincolare immediatamente il legislatore, ripercuotersi sulla interpretazione della legislazione precedente e sulla perdurante efficacia di alcune parti di questa; vi sono pure norme le quali fissano princìpi fondamentali, che anche essi si riverberano sull'intera legislazione. Pertanto è il contenuto concreto delle norme dettate nell'articolo 21 della Costituzione e il loro rapporto con le disposizioni dell'art. 113 della legge di p.s. che dovranno essere presi direttamente in esame, per accertare se vi sia contrasto dal quale derivi la illegittimità costituzionale di queste ultime disposizioni" (sentenza n. 1 del 1956).

É certo importantissimo affermare che possono darsi contrasti tra le norme legislative e le "norme [costituzionali] le quali fissano princìi fondamentali, che anche essi si riverberano sull'intera legislazione". Ma con ciò si resta pur sempre sul terreno del diritto scritto e della sua interpretazione. É nella metà degli anni Settanta che vengono invece poste le premesse della frattura madre, in quel tornante storico nel quale sembrò che tutta la cultura giuridica volesse partecipare al grande moto di attuazione della Costituzione: erano gli anni dell'approvazione dello Statuto dei Lavoratori e poi dell'istituzione del Servizio Sanitario Nazionale ..... La configurazione della Costituzione aperta al riconoscimento di diritti non scritti, come successivamente avvenne, segna la frattura decisiva: la Corte diventa interprete della realtà sociale, e crea un rapporto circolare in cui l'interpretazione della realtà sociale diventa 
la premessa dell'interpretazione della Costituzione, e dell'attivazione della fonte sulla produzione di cui all'art. 2. Tale riconoscimento avviene con diversi livelli di chiarezza e in modo non sempre esplicito, ma ricavabile dalla motivazione della decisione apparendo in taluni passaggi come presupposto: v., da ultimo, sent. n. 242 del 2019 (aiuto al suicidio), sent. n. 162 del 2014 (fecondazione eterologa), sent. n. 138 del 2010 (unione omosessuale). La sua conseguente azione regolatrice, che in tal modo si ridefinisce come sostanzialmente rappresentativa (riproponendo la storica distinzione tra rappresentanza ed elettività), assume un ruolo che ha condotto a renderla rilevante nella definizione della forma di governo. Il che, nel linguaggio costituzionalistico italiano, significava dire che la Corte ricopre un ruolo rilevante nello svolgimento della funzione di indirizzo politico tout court.

Dalla Costituzione intesa come testo che riceveva il suo significato, la sua unità e il suo indirizzo di sviluppo dalla Costituzione materiale, intesa come una interpretazione politica consensuale, data e stabilizzata, della società, che si esprimeva fondamentalmente attraverso il potere rappresentativo, si è passati a un'idea di Costituzione come un "tutto spirituale" capace di trasformare in diritto, a opera degli interpreti, le attese che la coscienza sociale del tempo fa emergere. Con una ulteriore distinzione: che altro è sostenere che la Costituzione scritta autorizza a includere in essa singoli diritti - che essa stessa non prevede - ma che sono forme stabilmente definite dalla coscienza sociale del tempo, e che in quanto tali devono essere recepite (siamo al confine scivoloso tra il diritto consuetudinario e il diritto scritto). Altro è invece sostenere che la Costituzione scritta autorizza a includere in essa singoli diritti - che essa stessa non prevede - come "essenze morali" o metafisiche, o come "valori" auto-evidenti. Qui siamo al confine tra diritto positivo e giusnaturalismo, o addirittura tra diritto positivo e "infatuazioni sentimentali”.

9. Ma fu una vera frattura? Nel diritto pubblico italiano è sempre stata presente una vena storicistica, che ha toccato il suo vertice teorico (non privo di ambiguità) con Santi Romano, e che la Scuola italiana del diritto pubblico ha mantenuto. Basti citare le parole pronunciate da V.E. Orlando davanti all'Assemblea Costituente, il 22 dicembre 1947, a Costituzione approvata, quando ribadì la sua fedeltà a quella scuola giu- 
ridica che concepisce il diritto come una "pianta, che mette nella terra le sue profonde radici, che [si] alimenta ... raccogliendo dall' aria, dalla luce, dalla profondità dell'humus le ragioni della sua esistenza".

La frattura di cui sin qui si è parlato costituisce in fondo il riemergere di questa concezione: lo spostamento del pendolo dal modo di intendere la Costituzione come disciplina determinata dalla sua origine storica, politica ed artificiale, polemica nei confronti del suo tempo, al modo di intenderla come disciplina che segue l'evoluzione della società.

Questo secondo modo di intendere la Costituzione è riconducibile alla concezione del diritto che è oggi difesa - con grandissima cultura - da Paolo Grossi: all'idea del diritto quale risultato di una invenzione. Secondo il Presidente emerito della Corte costituzionale, è necessario mettere a nudo "una verità, indubbiamente elementare ma anche indubbiamente elusa dalla stragrande maggioranza dei giuristi, tutti (o quasi) portatori di un intatto verbo normativistico, sempre meno intatto ma tuttavia perdurante, cioè duro a morire: il diritto quale risultato di una invenzione, percepito cioè non come qualcosa che si crea da parte del potere politico ma come qualcosa che si deve cercare e trovare (secondo il significato dello invenire latino) nelle radici di una civiltà, nel profondo della sua storia, nella identità più gelosa di una coscienza collettiva; e ne debbono essere inventori, fuori della vulgata corrente, in primo luogo i legislatori, ma, poi, anche i giuristi teorici e pratici nella loro complessa funzione. Di questa invenzione, che è un carattere particolarmente evidente nel diritto di questo nostro tempo di transizione e di mutazioni, le Costituzioni del secondo momento costituzionalistico - quello posweimariano, per intenderci - sono, a mio avviso, la testimonianza più espressiva" 3 .

Sia consentita una brevissima annotazione. Si è prima detto che lo sganciamento dei diritti soggettivi costituzionali dal diritto costituzionale oggettivo potrebbe essere spiegato - integrando la teoria del rinvio recettizio ex art. 2 Cost. - facendo leva sulla teoria del rinvio a fonti costituite da fatti normativi. Più precisamente: sul rinvio alla "necessità". Questa scelta ci incammina - con il suo massimo teorizzatore, Santi Romano - sulla scivolosa via del rapporto tra l'ordinamento statale e gli ordinamenti informali o allo stato diffuso: quegli ordinamenti

3 P. Grossi, L'invenzione del diritto, Bari-Roma, Laterza, 2017, p. X. 
che Romano chiama "embrionali" 4 , e tra questi quelli in cui si realizza l'organizzazione di interessi lato sensu politici fuori dallo Stato. Ci verremmo così a trovare nel clima culturale in cui il giovanissimo Romano, nel 1902, scriveva che la "necessità ... è la fonte prima del diritto, di quel diritto che scaturisce immediatamente e direttamente dalle forze sociali, in modo così categorico, esplicito, certo, da non permettere che tra i bisogni sociali stessi che determinano la norma giuridica e il rinvenimento e la dichiarazione di quest'ultima si frapponga l'attività razionale degli organi competenti a questa dichiarazione"s. La necessità, dunque, come fonte non bisognosa di riconoscimento. É qui inutile intrattenerci sul percorso che porterà Romano, nel 1946, con la II edizione de L'ordinamento giuridico, ad ammettere che il riconoscimento nell'ordinamento statale della validità di norme prodotte in ordinamenti diversi, necessita della legge.

L'espansione del potere giudiziario potrebbe essere intesa - al di là delle tante configurazioni che ne danno i diversi autori - come derivante da, e consistente in, una attività di "rinvenimento" (nel linguaggio romaniano ${ }^{6}$ ) di norme giuridiche extra legislative - appartenenti dunque ad ordinamenti altri - ad opera della giurisprudenza, innanzi tutto costituzionale (attraverso la lente dei, sottodefiniti, princìpi costituzionali) che le "dichiara" nei propri provvedimenti e che, con ciò, attribuisce loro rilevanza/validità nell' ordinamento interno. É una ricostruzione, questa, che colma una lacuna dell'impianto romaniano incardinando nella giurisprudenza la funzione del "riconoscimento". Evita altresì di avventurarsi sulle sabbie mobili del neo-giusnaturalismo o dell'iperpositivismo dei princìpi/valori e vede nei diritti emersi dalla "coscienza sociale" e privi di un solido appiglio nella legislazione ordinaria, un

4 S. Romano, L'ordinamento giuridico, II ed, Firenze, Sansoni, 1946, ora in "L' 'ultimo' Santi Romano", Milano, Giuffrè, 2013, con nota bio-bibliografica a cura di Alberto Romano, p. 27, nota 29 ter.

5 S. Romano, Osservazioni preliminari per una teoria sui limiti della funzione legislativa nel diritto italiano, in "Scritti minori", I, Milano, Giuffrè, 1990 (rist. ed. 1950), p. 217 sgg..

6 S. Romano, Osservazioni preliminari per una teoria sui limiti della funzione legislativa nel diritto italiano, cit., p. 236. Sul punto, si veda M. Dogliani La fortuna della teoria romaniana dell'ordinamento nelle varie aree disciplinari: diritto costituzionale, in "Diritto Pubblico", n. 3, 2018.

7 S. Romano, Osservazioni preliminari per una teoria sui limiti della funzione legislativa nel diritto italiano, cit., p. 236. 
diritto di origine extrastatale rinvenuto e reso valido dalla giurisprudenza (il nucleo fondamentale della frattura). Queste considerazioni collocano la questione della "invenzione" di cui parla Grossi sul piano della cultura giuridica e della elaborazione che precede la produzione del diritto ad opera delle fonti autorizzate, mentre la discussione qui verte solo sulla produzione di diritto "valido" ad opera di fonti non autorizzate (la giurisprudenza costituzionale). In altre parole: la giurisprudenza, nella misura in cui non pone a proprio fondamento l'interpretazione di una disposizione scritta, può essere considerata una fonte del diritto, un caso di "invenzione" del diritto non creato dal potere politico? In caso di risposta affermativa la questione diventa quella di decidere se questa funzione di rinvenimento debba essere concentrata nella giurisprudenza costituzionale, al fine di evitarne gli eccessi e assicurarne effetti erga omnes, o possa essere estesa alla giurisprudenza ordinaria (per vie quali l'interpretazione conforme che assuma la veste di interpretatio abrogans, il ricorso alla Corte di Lussemburgo o di Strasburgo ...). E' certo infatti che tale giurisprudenza ordinaria può raggiungere punte propriamente "eversive" 8 : fenomeno neanche troppo limitato9.

\footnotetext{
Abstract - Through an unusual category for constitutional theory - disruption - this short essay deals with the introduction of constitutional justice with the Constitutional Court in Italian Constitution.

From a historiographical point of view the essay describes the most important events when the Court has defined the fea-
}

tures and the limits of the review of legislation in Italy, and so its instruments and position and the position of Parliament and judges as an effect.

The paper points out that the most important disruption has been the disjunction of subjective rigths from law, above all through art. 2 of the Constitution.

8 C. Pinelli, Intervento al dibattito su "Giudici e legislatori" in "Diritto Pubblico", n. 2, 2016, p. 483.

9 A. Travi, Intervento al dibattito su "Giudici e legislatori" in "Diritto Pubblico", $\mathrm{n}$. 2, 2016, p. 504: "La vicenda "Stamina" ... si è risolta in un numero elevato di pronunce di giudici di merito (ben più di un centinaio) che hanno imposto un trattamento non consentito dalla legge. ... lo strumento del ricorso previsto dall'art. 700 c.p.c. è stato utilizzato per una disapplicazione della legge, in moltissimi di questi casi senza alcun rinvio alla Corte costituzionale". 